\title{
State-LeVEL COVID-19 TREnd Forecasting Us- ING MOBILITY AND POLICY DATA
}

\author{
Yifei Wang ${ }^{+}, \mathrm{Hao}$ (Michael) Peng*, Long Sha ${ }^{+}$, Zheyuan Liu ${ }^{+}$, Pengyu Hong ${ }^{+}$ \\ +Department of Computer Science \\ Brandeis University \\ Waltham, MA 02453, USA \\ \{yifeiwang, longsha, zheyuanliu, hongpeng\}@brandeis.edu \\ *Andover High School \\ 80 Shawsheen Rd. \\ Andover, MA 01810 \\ michael@mcmoo.org
}

\begin{abstract}
The importance of pandemic forecast cannot be overemphasized. We propose an interpretable machine learning approach for forecasting pandemic transmission rates by utilizing local mobility statistics and government policies. A calibration step is introduced to deal with time-varying relationships between transmission rates and predictors. Experimental results demonstrate that our approach is able to make accurate two-week ahead predictions of the state-level COVID-19 infection trends in the US. Moreover, the models trained by our approach offer insights into the spread of COVID-19, such as the association between the baseline transmission rate and the state-level demographics, the effectiveness of local policies in reducing COVID-19 infections, and so on. This work provides a good understanding of COVID-19 evolution with respect to state-level characteristics and can potentially inform local policymakers in devising customized response strategies.
\end{abstract}

\section{INTRODUCTION}

The novel coronavirus disease 2019 (COVID-19) has caused a global pandemic (Zhu et al., 2020) and imposes unprecedented challenges on governments and societies around the world. The COVID-19 outbreak has two key features: high covertness and high transmissibility (Hao et al. 2020), which have pushed some healthcare systems to the brink of collapse and have prompted governments to impose strict policies on physical isolation and travel restrictions so as to mitigate the spread of COVID-19. It is hence essential to accurately predict the spread of COVID-19. Such research, especially research on local trend forecasting, can provide valuable insights to help local authorities prepare their health systems and deploy appropriate policies to mitigate the spread.

Conventional mathematical modelling of infectious diseases in epidemiology, such as compartmental models (Kermack \& McKendrick, 1927) and their derivatives (Hao et al., 2020, Croccolo \& Roman 2020; Palladino et al., 2020), have been used to reconstruct and forecast transmission dynamics at macro levels. Such a model usually builds an ODE system in a top-down way to approximate the epidemic process and estimate model parameters via Monte Carlo methods. Accurate domain knowledge is required to design appropriate compartments as well as their relationships.

It is highly desired to develop models that not only capture dynamic relationships between infectious data and population but also make accurate forecasts on the spread of the disease. Oliver et al. (2020) argued that human behavior, especially mobility and physical co-presence, was necessary for spread analysis during all stages of a pandemic life cycle. Thanks to the pervasive mobile devices, in-time mobility statistics can now be obtained at a large scale. In fact, mobility information had been successfully used in building epidemiological models for H1N1 flu outbreaks (Balcan et al., 2009). This work estimates the reproduction number based on high-quality population mobility patterns, so as to make up missing incidence data during the early phase of the H1N1 pandemic. It was able to 
uncover the seasonal transmission potential of H1N1 in affected countries at the early stage, using mobility and transportation data worldwide in addition to the raw count of cases.

Present work. In this work, we tackle the problem of forecasting the state-level daily new cases of COVID-19 in the US. We have developed a machine learning approach to estimate the state-level daily transmission rates via robust regression on local mobility statistics and government policies. The predicted daily transmission rates can then be accumulated to estimate the daily new cases. There are temporal variances in population behaviors (e.g., awareness of conditions relating to public health, compliance to policies, etc.), which, if not considered, can greatly affect the performance of our approach. To deal with this problem, we added a novel calibration step to our modeling, which assumes the relationships between the transmission rate variable and its predictors remain unchanged within a short time window. Empirical studies show that our approach can make satisfying predictions two weeks into the future for most states. Furthermore, our approach is well interpretable and offers insights into the spread of this pandemic. For example, we show that the baseline transmission rate, which is indicated as the bias terms in our trained models, is highly associated with state-level demographics. In addition, the factors identified to be significant in making predictions are quite consistent across states with how people and governments fight against COVID-19.

\section{RELATED WORK}

Previous works on COVID-19 trend prediction can be roughly categorized into the following two types.

Compartmental models. Most recent approaches for COVID-19 spread analysis in epidemiology are derivatives of the Susceptible Infectious Recovered (SIR) model (Kermack \& McKendrick, 1927, Harko et al. 2014), a widely used compartmental model. These approaches group the subjects in the system of interest into different population compartments when modeling epidemic spread. The dynamics of the system is characterized by the transitions of subjects between compartments, which are mathematically expressed as a set of differential equations. Croccolo \& Roman (2020) extended the SIR model to encompass the effects of lockdown policy and applies it to COVID-19 in the US. Palladino et al. (2020) improved the standard SIR model to have a varying diffusion velocity of virus, which accounts for nonpharmaceutical interventions, and applied the model to COVID-19 in Italy. In another work, Hao et al. (2020) proposed a SAPHIRE model that contains seven compartments (susceptible, exposed, presymptomatic infectious, ascertained infectious, unascertained infectious, isolation in hospital and removed) to reconstruct transmission dynamics of COVID-19 in Wuhan, China between 01/01/2020 and 08/03/2020. This time period was divided into 5 segments (Pan et al. 2020), in each of which, the ascertainment rate and transmission rate were assumed to be fixed. They also assumed a constant population size and a constant number of travellers in each period. Fernández-Villaverde \& Jones (2020) incorporated social distancing into a SIRD (SusceptibleInfectious-Recovered-Dead) model that allows a time-varying contact rate so as to capture changes associated with social distancing and quarantine policy. They conducted simulations of deaths on various regions, such as New York City, Italy, Sweden and Spain. Picchiotti et al. (2020) built a SEIR (Susceptible-Exposed-Infected-Recovered) model that considers both personal protective measures and mobility restrictions represented as decreasing logistic functions. Chang et al. (2020) introduced a metapopulation SEIR model that integrated fine-grained, dynamic mobility networks to simulate the spread of SARS-CoV-2 in 10 of the largest US metropolitan statistical areas. The IHME COVID-19 Forecasting Team (2020) proposed a deterministic SEIR framework to model possible trajectories of COVID-19 infections and the effects of non-pharmaceutical interventions in the US at the state level. Dandekar \& Barbastathis (2020) augmented the SIR model to include a time varying quarantine strength term, which is learned by a neural network from real data. Yang et al. (2020b) compared a set of SIR based models (e.g., SIR, SEIR, SEIR-AHQ (Tang et al. 2020), SEIR-QD (Peng et al., 2020), SEIR-PO (proposed), etc.) on their forecast abilities using the daily reported confirmed infected case data from the China CDC. It is evident that most compartmental models focus on dynamics reconstruction and lack the ability to make long-term predictions.

Machine learning models. Machine learning approaches are very capable of learning complex dynamic patterns and relationships directly from data. Punn et al. (2020) and Tuli et al. (2020) applied machine learning techniques (e.g., support vector regression, polynomial regression, robust 


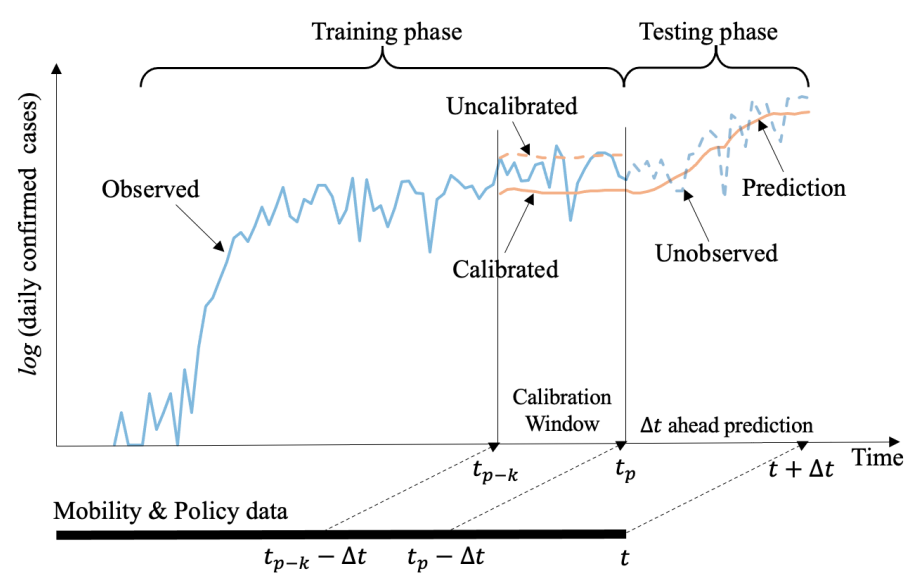

Figure 1: Illustration of the proposed approach for predicting the state-level spread of COVID19. The $x$-axis represent time. The $y$-axis represents the number of daily cases in logarithmic scale. Our approach makes $\Delta t$-ahead predictions, starting at time $t_{p}$, using the state-level daily mobility statistics and policies. The model calibration is done using the daily confirmed case data within the time window $\left[t_{p-k}, t_{p-1}\right]$.

Weibull fitting, etc.) to fit the nationwide epidemic curve without considering any exogenous factors. Yang et al. (2020a) proposed a GRU-based framework for state-level trend prediction, integrating the time-varying epidemic information with environmental factors. This work incorporated static external factors including local population and age structure while ignoring dynamic population behaviors. Kapoor et al. (2020) developed a GNN-based approach for county-level COVID-19 forecasting, where Google's human mobility data across all counties in the US are represented as a single large-scale spatio-temporal graph. Nevertheless, it did not consider other significant external factors (e.g., mandatory or voluntary mask policies). Ramchandani et al.(2020) divided county-level weekly rises of confirmed COVID-19 cases into 4 coarse categories and developed DeepCOVIDNet based on the DeepFM (Guo et al., 2017) framework that used the demographic statistics and crosscounty mobility data provided by $S$ afeGraph to make coarse-level predictions.

\section{RESUlts}

We applied our approach to predict the state-level infection trends of COVID-19 in the US. The results reveal the interaction between the spread of COVID-19 and the state-level mobility factors and restriction policies. In addition, we show that our approach learns the "bias" linked to state-level demographic characteristics.

\subsection{State-LeVel Epidemic Forecasting Model}

Our approach (Figure 1) trains a model in a pure data-driven manner for each state in the US, including the District of Columbia (DC), to make $\Delta t$-ahead prediction of the state-level daily transmission rate $\hat{r}_{t+\Delta t}$ using the state-level daily mobility statistics and government policies at time $t$. The estimated daily confirmed cases in this state can then be derived from the corresponding estimated $\hat{r}_{t}$ in an accumulated manner. Furthermore, a calibration step is proposed to adjust for short-term changes in population behaviors. We abuse the term "state" a little bit to indicate a state or the DC throughout the paper. The hyper-parameter $\Delta t$ specifies how far in the future a prediction is made, and is automatically adjusted for each state. The detailed description of the model is provided in Appendix $\mathrm{A}$. The following lists the data used in this work:

- COVID-19 daily confirmed case data: The latest COVID-19 daily cases data was obtained from The New York Times!

1 https://github.com/nytimes/covid-19-data 
Preprint.

- State-level mobility data: The trip-by-distance mobility data is made available by the Maryland Transportation Institute and Center for Advanced Transportation Technology Laboratory at the University of Maryland ${ }^{2}$. The daily trips are grouped into 10 categories based on the travel distances and another Staying-at-home category indicates the ratio of population mostly at home.

- State restriction policy: The information about state-level restrictions were extracted from "The Coronavirus Outbreak" forum on the New York Times 3 . This work considers the mask policy and the restaurant restriction policy.

- State-level demographic information: This data includes the state-level population density information published by the U.S. Census Burea ${ }^{4}$ the race structure information (fraction of 7 different race categories) collected by the COVID Tracking Projec 5 , and the age structure information (fraction of 6 non-overlapping age groups as well as the high risk population) collected by the Kaiser Family Foundation 6

The whole dataset contains the daily confirmed cases $(01 / 21 / 2020$ - 12/08/2020) in the 51 states of the US. The data was split into a training set $(01 / 21 / 2020-11 / 24 / 2020)$ and a test set $(11 / 25 / 2020$ - 12/08/2020). Fifty states issued the restaurant policies, and 34 states issued the public mask policies. For each state, we fit a model using the train data starting from its pandemic start date to 11/24/2020. The pandemic start date of a state is decided in the way discussed in Section A.1. The only hyper-parameter of the model is $\Delta t$, which is related to the incubation time of COVID-19 and the efficiency of a state's healthcare system. The typical incubation period for COVID-19 is around 14 days according to the CDC. Since there were delays in taking tests and reporting cases, we limited $\Delta t$ to between 15 and 20, and applied ten-fold cross-validation using the training data to determine the optimal $\Delta t$ of each state. In the test phase, we first smoothed the predicted transmission rate, $\log \hat{r}_{t}$, by taking an exponential moving average on the previous 3 days. Then we conduct the calibration step (see Section A.3 using the data between 11/18/2020 and 11/24/2020.

\subsection{PREDICTION EVALUATION METRICS}

We evaluate the prediction performance based on normalized RMSE (nRMSE):

$$
\mathrm{nRMSE}=\frac{\operatorname{RMSE}\left(\log \widehat{D}_{\left[t_{p}: t_{p}+m\right]}, \log D_{\left[t_{p}: t_{p}+m\right]}\right)}{\operatorname{Median}\left(\log D_{\left[t_{p}: t_{p}+m\right]}\right)}
$$

where the prediction period is $\left[t_{p}, t_{p}+m\right], m$ is set to be no more than 14 in this work, $\log D_{\left[t_{p}: t_{p}+k\right]}$ indicates the logarithm of the daily confirmed cases between $t_{p}$ and $t_{p}+m$, and $\log \widehat{D}_{\left[t_{p}: t_{e}+m\right]}$ indicates the logarithm of the predicted daily confirmed cases. nRMSE estimates the relative deviation from the the local COVID-19 trend. A value of 0.01 represents the average prediction deviates $1 \%$ from the true local trend in a logarithmic scale. We also report the relative accumulated log error (RALE) of cases during $\left[t_{p}, t_{p}+m\right]$ :

$$
\operatorname{RALE}=\frac{\left|\sum\left(\log \widehat{D}_{\left[t_{p}: t_{p}+m\right]}-\log D_{\left[t_{p}: t_{p}+m\right]}\right)\right|}{\sum\left(\log D_{\left[t_{p}: t_{p}+m\right]}\right)}
$$

RALE captures the relative deviation from the accumulated cases within $m$ days. A value of 0.01 represents the predictive cases deviates $1 \%$ from the true cases within $m$ days in a logarithmic scale.

\footnotetext{
2 https://data.bts.gov/Research-and-Statistics/Trips-by-Distance/ w96p-f2qv html

https://www. census.gov

5 https://covidtracking.com

$\epsilon$ https://www.kff.org/other/state-indicator/distribution-by-age/ ? current Timeframe $=0$ \& sortModel $=\div 7 \mathrm{~B} \div 22 \operatorname{col} I d \div 22: \div 22$ Location $\div 22, \div 22$ sort $\div 22$ : $\div 22 \mathrm{asc} \div 22 \% 7 \mathrm{D} \div 23$ notes
} 


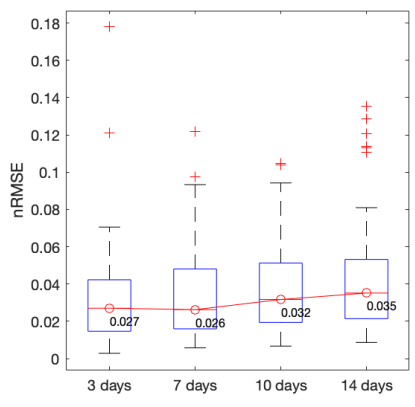

(a) nRMSE

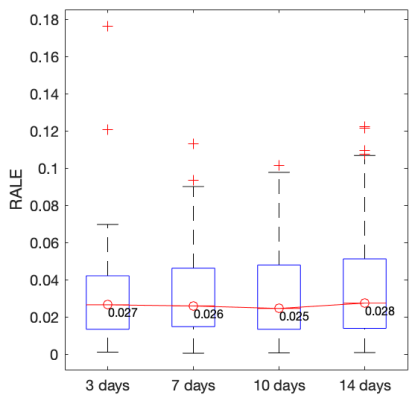

(b) RALE

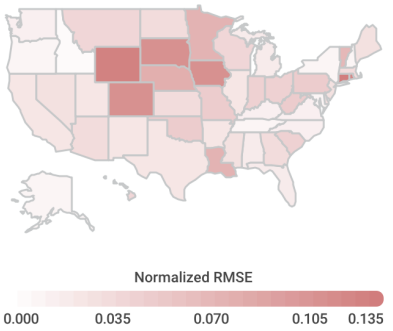

(c) Heatmap of nRMSE.

Figure 2: The summary of the 3-, 7-, 10- and 14-day state-level forecasting performance. (a) The state-level nRMSE values. (b) The state-level RALE values. (c) The geographic heatmap of the 14-day forecasting nRMSE.

\subsubsection{SUMMARY OF THE PREDICTION PERFORMANCE}

We ran our approach to make the 3-, 7-, 10- and 14-day predictions in all states. The results are summarized in Figure 2 with details in Table 2 The median nRMSE for 14-day forecasting is 0.035 (i.e., the prediction deviates $\approx 3.5 \%$ from the real local trend at a logarithmic scale). Most nRMSEs of the 14-day forecasting results are within 0.05 , indicating that our model works well for most states in the US. The RALE results show a similar trend. We observe that both nRMSE and RALS increase slightly with the forecasting time extends. For instance, the median values of the 3-and 14-day nRMSEs are 0.027 and 0.035 , respectively. Figure 3 visualizes the 14 -day predictions (both the transmission rates and the daily confirmed cases) of two states, NY (nRMSE $=0.0105$, RALE $=0.0036)$ and LA $(\mathrm{nRMSE}=0.0755, \mathrm{RALE}=0.0710)$. The prediction results on NY and LA are among the best and worst, respectively.

We should point out that a large nRMSE or RALE may indicate large volatility in the confirmed daily cases due to delay in reporting, rather than the weak performance of the corresponding model. Figure 10 shows four representative states that contain significant volatilities in daily confirmed cases in or after the forecasting period $(11 / 25 / 2020-12 / 08 / 2020)$. However, their overall future trends match our forecasting curves very well.

\subsection{SignificANT FACtors IN PREDICTING COVID-19 TREND}

The predictors have different levels of effects on COVID-19 trend prediction across the US (see Figure $4 \mathrm{~b}$-f, with more details in Figure 8). Using 0.05 as the $p$-value cutoff, we observed several factors are frequently identified as significant across the states (Figure 4a). Mask Policy has the highest frequency of 0.7647 ( 26 out of 34 states that issued the mask policy), indicating that the mask policy has the most impact on the change of epidemic dynamics. The estimated coefficients of Mask Policy and three other significant factors (Restaurant Policy, Stay-at-home and Dis-0-1) are negative in nearly all cases (Appendix B), which indicates these factors help hamper the spread of COVID-19. This is consistent with how people and governments fight against COVID-19: wearing masks, closing restaurants and staying at home. Mobility categories Dis-1-3 and Dis $>500$ also have general impacts. However, their estimated coefficients are positive in most cases, indicating that they help promote the spread of COVID-19. We suspect that Dis-1-3 may correspond to walking within local communities and that Dis $>500$ mostly represents cross-state travels.

Interestingly, other mobility categories become significant for a few states. For example, the long distance mobility categories (Dis-250-500 and Dis $>500$ ) are both significant in regression for states NV and MT. This might be explained by the low population densities in those states. There may be other very complex interactions between mobility and demographic properties, which we leave for future exploration. 

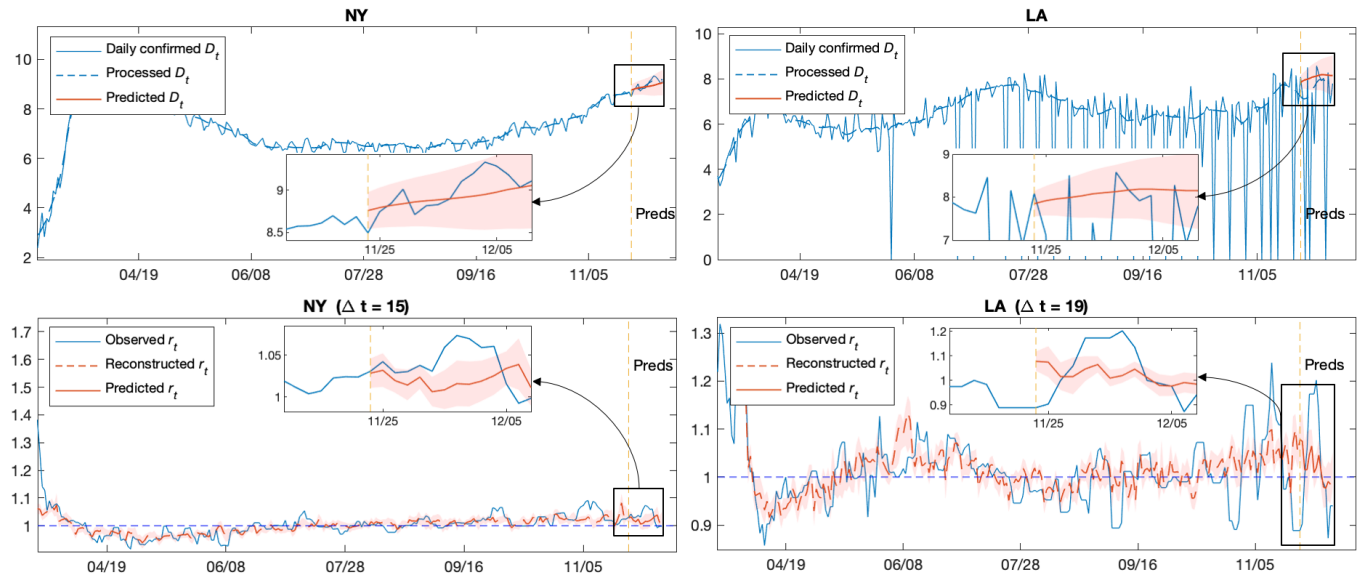

Figure 3: The 14-day predictions of the COVID-19 transmission rates and daily cases in NY $(\mathrm{nRMSE}=0.0105, \mathrm{RALE}=0.0036)$ and LA $(\mathrm{nRMSE}=0.0755, \mathrm{RALE}=0.0710)$ in logarithmic scale. All $x$-axis indicate time. The $y$-axes in the top plots indicate the logarithm of the daily confirmed cases. The $y$-axes in the bottom plots indicate the transmission rate values. The yellow dash vertical lines indicate the starting points of the prediction periods. The blowouts highlight the predictions. The red shaded areas indicate the $95 \%$ confidence intervals.

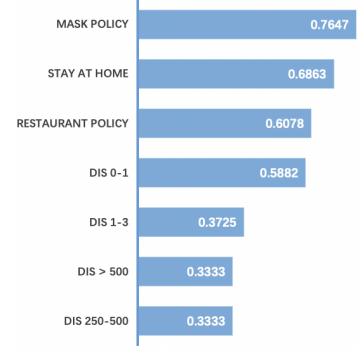

(a) Frequency of significant factors

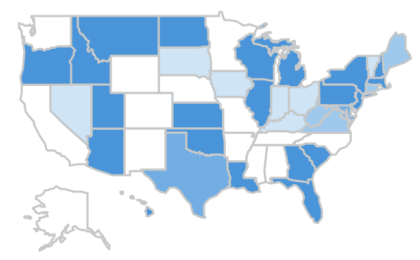

(d) Stay-at-home

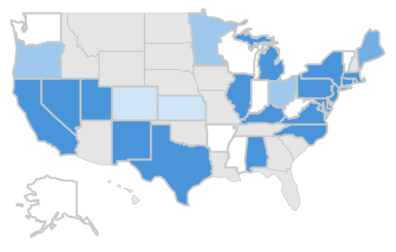

(b) Mask Policy

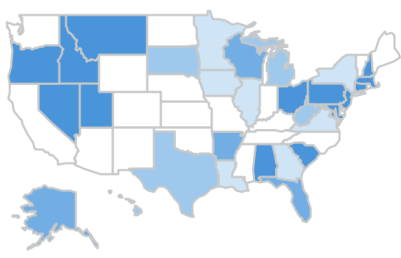

(e) Dis-0-1

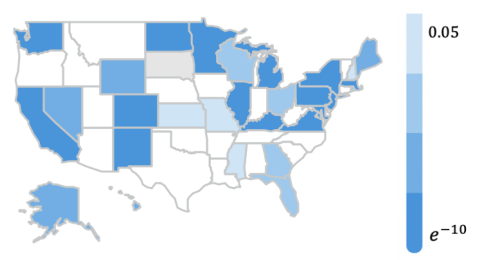

(c) Restaurant Policy

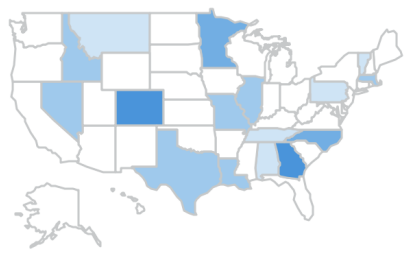

(f) Dis $>500$

Figure 4: (a) Frequency of each factor identified to be significant within states. Factor with high frequency implies its general influences on most states. (b)-(f) Heatmaps of each factor's p-values. Here a state is colored grey if it doesn't incorporate such factor into regression. Remaining results are also reported in Figure 8 in the Appendix.

\subsection{DEMOGRAPHIC INTERPRETATION OF THE STATE-LEVEL BIASES IN COVID-19 TRANSMISSION}

We trained one daily case prediction model for each state (including the DC) and obtained 51 models in total. Notice that the intercept term in each model represents the baseline transmission rate of the corresponding state. We hypothesized that the differences in the baseline transmission rates were due to the state-level demographics (e.g., population density, age structure, race structure, etc.). 


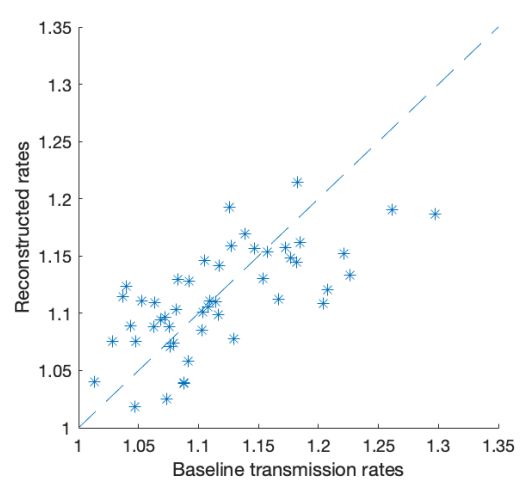

Figure 5: Daily transmission rate is estimated by time-dependent mobility variables as well as categorical variables for policies. Then daily cases are predicted from previous transmission rates. The biased term (intercept) from the regression model is the baseline transmission rate under normal mobility and conditions before the pandemic. The baseline rate is highly related to each state's demographics.

To investigate this, we used lasso (Tibshirani, 1996) to select four demographic variables highly related to the state-level model biases, which include Top Densit) ${ }^{7}(p$-value $=0.0293)$, Adults-35-54 $(p$-value $=0.1003)$, Hispanic-Or-Latino $(p$-value $=0.0112)$ and AmericanIndian-Or-AlaskaNative $(p$-value $=0.1507)$. We then used them to reconstruct the baseline transmission rates using nonregularized linear regression (see Table 1 and Figure 5). Our findings resonate with the findings in previous works that the spread of COVID-19 were highly relevant to population densities (Rocklöv \& Sjödin, 2020) and ethnic minorities (Dyer, 2020, Kirby, 2020), and hence aid in understanding the spread of COVID-19 and increase the interpretability of our model.

\section{CONCLUSIONS}

In this paper, we propose a data-driven approach that trains regression models for forecasting the state-level COVID-19 daily transmission rates using the state-level mobility data and restrictive policies. The transmission rates can then be used to estimate the daily confirmed cases in an accumulated manner. Our approach uses a calibration step to adjust for short-term changes in population behaviors. Our empirical study results show that the proposed approach can reliably and accurately forecast ( 2 weeks ahead) the state-level COVID-19 spread. We also studied statistically significant factors as well as their impacts on the COVID-19 pandemic, and the findings allow us to better understand how population mobility and government policies may affect the spread of COVID- 19 . Our prediction results can be used by local governments or healthcare systems to prepare ahead, and the discovered quantitative relationships between COVID-19 and population mobility as well as policies can be used to by policymakers in devising customized response strategies.

\section{Code AND Data AVAilability}

Our codes and the data used in this work are available on GitHub at https://github.com/ yifeiwang15/COVID-19-Mobility.

\section{AUTHOR CONTRIBUTIONS}

Yifei Wang developed the whole model architecture, planned and carried out the experiments, and mainly wrote the manuscript. Pengyu Hong initialized this project, conceived of the presented idea, supervised the experiments and revised the manuscript. Michael Peng did data extraction as well as data filtering and helped improve the manuscript. Michael Peng and Frank Liu developed a website (https://broad-well.github.io/covid-trend-prediction/) to visualize our predictions on all states. Long Sha participated in brainstorms and discussions.

\footnotetext{
${ }^{7}$ The highest population density within state.
} 
Preprint.

\section{ACKNOWLEDGMENTS}

This work was partially supported by NSF OAC 1920147.

\section{COMPETING INTERESTS STATEMENT}

The authors declare no competing interest.

\section{REFERENCES}

Duygu Balcan, Hao Hu, Bruno Goncalves, Paolo Bajardi, Chiara Poletto, Jose J Ramasco, Daniela Paolotti, Nicola Perra, Michele Tizzoni, Wouter Van den Broeck, et al. Seasonal transmission potential and activity peaks of the new influenza a $(\mathrm{h} 1 \mathrm{n} 1)$ : a monte carlo likelihood analysis based on human mobility. BMC medicine, 7(1):45, 2009.

Serina Chang, Emma Pierson, Pang Wei Koh, Jaline Gerardin, Beth Redbird, David Grusky, and Jure Leskovec. Mobility network models of covid-19 explain inequities and inform reopening. Nature, pp. 1-6, 2020.

Fabrizio Croccolo and H Eduardo Roman. Spreading of infections on random graphs: A percolationtype model for covid-19. Chaos, Solitons \& Fractals, 139:110077, 2020.

Raj Dandekar and George Barbastathis. Quantifying the effect of quarantine control in covid-19 infectious spread using machine learning. medRxiv, 2020.

Owen Dyer. Covid-19: Black people and other minorities are hardest hit in us, 2020.

Chao Fan, Sanghyeon Lee, Yang Yang, Bora Oztekin, Qingchun Li, and Ali Mostafavi. Effects of population co-location reduction on cross-county transmission risk of covid-19 in the united states. arXiv preprint arXiv:2006.01054, 2020.

Jesús Fernández-Villaverde and Charles I Jones. Estimating and simulating a sird model of covid-19 for many countries, states, and cities. Technical report, National Bureau of Economic Research, 2020.

Huifeng Guo, Ruiming Tang, Yunming Ye, Zhenguo Li, and Xiuqiang He. Deepfm: a factorizationmachine based neural network for ctr prediction. arXiv preprint arXiv:1703.04247, 2017.

Xingjie Hao, Shanshan Cheng, Degang Wu, Tangchun Wu, Xihong Lin, and Chaolong Wang. Reconstruction of the full transmission dynamics of covid-19 in wuhan. Nature, 584(7821):420-424, 2020.

Tiberiu Harko, Francisco SN Lobo, and MK Mak. Exact analytical solutions of the susceptibleinfected-recovered (sir) epidemic model and of the sir model with equal death and birth rates. Applied Mathematics and Computation, 236:184-194, 2014.

Paul W Holland and Roy E Welsch. Robust regression using iteratively reweighted least-squares. Communications in Statistics-theory and Methods, 6(9):813-827, 1977.

Amol Kapoor, Xue Ben, Luyang Liu, Bryan Perozzi, Matt Barnes, Martin Blais, and Shawn O'Banion. Examining covid-19 forecasting using spatio-temporal graph neural networks. arXiv preprint arXiv:2007.03113, 2020.

William Ogilvy Kermack and Anderson G McKendrick. A contribution to the mathematical theory of epidemics. Proceedings of the royal society of london. Series A, Containing papers of a mathematical and physical character, 115(772):700-721, 1927.

Tony Kirby. Evidence mounts on the disproportionate effect of covid-19 on ethnic minorities. The Lancet Respiratory Medicine, 8(6):547-548, 2020.

Nuria Oliver, Bruno Lepri, Harald Sterly, Renaud Lambiotte, Sébastien Deletaille, Marco De Nadai, Emmanuel Letouzé, Albert Ali Salah, Richard Benjamins, Ciro Cattuto, et al. Mobile phone data for informing public health actions across the covid-19 pandemic life cycle, 2020. 
Preprint.

Andrea Palladino, Vincenzo Nardelli, Luigi Giuseppe Atzeni, Nane Cantatore, Maddalena Cataldo, Fabrizio Croccolo, Nicolas Estrada, and Antonio Tombolini. Modelling the spread of covid19 in italy using a revised version of the sir model. arXiv preprint arXiv:2005.08724, 2020.

An Pan, Li Liu, Chaolong Wang, Huan Guo, Xingjie Hao, Qi Wang, Jiao Huang, Na He, Hongjie $\mathrm{Yu}$, Xihong Lin, et al. Association of public health interventions with the epidemiology of the covid-19 outbreak in wuhan, china. Jama, 323(19):1915-1923, 2020.

Liangrong Peng, Wuyue Yang, Dongyan Zhang, Changjing Zhuge, and Liu Hong. Epidemic analysis of covid-19 in china by dynamical modeling. arXiv preprint arXiv:2002.06563, 2020.

Nicola Picchiotti, Monica Salvioli, Elena Zanardini, and Francesco Missale. Covid-19 pandemic: a mobility-dependent seir model with undetected cases in italy, europe and us. arXiv preprint arXiv:2005.08882, 2020.

Narinder Singh Punn, Sanjay Kumar Sonbhadra, and Sonali Agarwal. Covid-19 epidemic analysis using machine learning and deep learning algorithms. medRxiv, 2020.

Ankit Ramchandani, Chao Fan, and Ali Mostafavi. Deepcovidnet: An interpretable deep learning model for predictive surveillance of covid-19 using heterogeneous features and their interactions. IEEE Access, 8:159915-159930, 2020.

Joacim Rocklöv and Henrik Sjödin. High population densities catalyse the spread of covid-19. Journal of travel medicine, 27(3):taaa038, 2020.

SafeGraph. Places schema. [EB/OL]. Available: https://docs.safegraph.com/docs/ places-schema\# Accessed Jul. 26, 2020.

Biao Tang, Xia Wang, Qian Li, Nicola Luigi Bragazzi, Sanyi Tang, Yanni Xiao, and Jianhong Wu. Estimation of the transmission risk of the 2019-ncov and its implication for public health interventions. Journal of clinical medicine, 9(2):462, 2020.

IHME COVID-19 Forecasting Team. Modeling covid-19 scenarios for the united states. Nature Medicine, 2020.

Robert Tibshirani. Regression shrinkage and selection via the lasso. Journal of the Royal Statistical Society: Series B (Methodological), 58(1):267-288, 1996.

Shreshth Tuli, Shikhar Tuli, Rakesh Tuli, and Sukhpal Singh Gill. Predicting the growth and trend of covid-19 pandemic using machine learning and cloud computing. Internet of Things, pp. 100222, 2020.

Tong Yang, Long Sha, Justin Li, and Pengyu Hong. A deep learning approach for covid-19 trend prediction. arXiv preprint arXiv:2008.05644, 2020a.

Wuyue Yang, Dongyan Zhang, Liangrong Peng, Changjing Zhuge, and Liu Hong. Rational evaluation of various epidemic models based on the covid-19 data of china. arXiv preprint arXiv:2003.05666, 2020b.

Na Zhu, Dingyu Zhang, Wenling Wang, Xingwang Li, Bo Yang, Jingdong Song, Xiang Zhao, Baoying Huang, Weifeng Shi, Roujian Lu, et al. A novel coronavirus from patients with pneumonia in china, 2019. New England Journal of Medicine, 2020.

\section{A Methods}

\section{A.1 DATA AND PREPROCESSING}

COVID-19 daily case data. The COVID-19 data used in this work is the US state-level daily confirmed cases, denoted as $D_{t}$ where $t$ is date. The data is very noisy, especially in the beginning of the pandemic, due to various reasons, such as, delay in reporting, and so on. We performed the following preprocessing. For each state, we first detected the pandemic start time $t_{s}$ as the first day of the first three consecutive days with non-zero daily confirmed cases. We then smoothed $D_{t}$ after 
$t_{s}$ by taking a moving median using a sliding window of size 7 followed by a moving average using a sliding window of size 5 . In the rest of the paper, $D_{t}$ refers to the preprocessed daily cases.

Mobility data. The state-level travel statistics are daily aggregates of residents' movements based on their mobile phone data, and provide information about population mobility. A trip was counted if a person stayed away from home for more than 10 minutes. The daily trips were grouped into 11 categories based on their travel distances (see Figure 6 for an example). For instance, the Dis5-10 category indicates the number of trips within the range of 5-10 miles. The Staying-at-home category records the size of the population that did not stay away from home for more than 10 minutes. In each state, the Staying-at-home category data was normalized by the state population, and other categories were normalized by the state population not staying at home. Each category is then standardized to represent the relative changes in mobility from the pre-pandemic level. This was done by subtracting the median pre-pandemic mobility value and then dividing the maximal pre-pandemic mobility value.

There exists abnormal activities across the US around the later summer 2020 when schools start and in early November 2020 when the election was held. These sudden irregular travel patterns were associated with distinct yet unknown population behaviors. We suspect that the subpopulation, which exerted the abnormal travel patterns, deployed special the required protection/quarantine means and hence contributed little to the spread of COVID-19. Hence, the corresponding mobility data should not be used without appropriate processing in training the models and making predictions. To this end, we detected outliers as samples more than three scaled median absolute deviations away from the median. Then, we conducted Principal Component Analysis on the training data and reconstructed the detected outliers with the first 4 principle components. Figure 7 shows an example of outlier detection and reconstruction in the mobility data category Dis $>500$ of the New York and California states.

State restriction policy. We considered the state-level mask wearing policy and restaurant opening policy as two binary variables. If a policy is instated, the value of its variable is 1 , otherwise 0 .

Demographic information. The following state-level demographic information, which was also used in Yang et al. (2020a): (i) local population density, (ii) local age structure (non-overlapping age groups), (iii) local race structure (different race categories).

\section{A.2 REgRESSION MODEL FOR EPIDEMIC PREDICTION}

The epidemic transmission rate in each state at time $t$ is defined as the ratio between the state-level confirmed daily cases at $t$ and that at $t-1$, i.e., $r_{t}=D_{t} / D_{t-1}$, where $D_{t}$ is the number of the daily confirmed cases at time $t$. The transmission rate $r_{t}$ can be algebraically mapped to the reproduction number in epidemiology (Fan et al. 2020). Assuming no auto-correlation in transmission rates, we first use a robust linear regression technique (Holland \& Welsch. 1977) to estimate the logarithm of the state-level transmission rate (i.e., $\log \hat{r}_{t+\Delta t}$ ) using the mobility statistics and policies at time $t$, where the hyper-parameter $\Delta t$ specifies how far in the future a prediction is made and its optimal value can be adjusted using ten-fold cross-validation. Assuming prediction starts at time $t_{p}$, the logarithm of the predicted daily cases $\hat{D}_{t+m}$, where $m \leq \Delta t$, can be derived using the estimated transmission rates as follows:

$$
\log \hat{D}_{t_{p}+m}=\log D_{t_{p}-1}+\sum_{k=1}^{m} \log \hat{r}_{t+k}
$$

where $D_{t_{p}-1}$ is the ground-truth number of the daily cases at time $t_{p}-1$.

\section{A.3 Calibrating the forecast}

The above regression model assumes stationary relationships between the transmission rate variable and the predictors (i.e., population mobility and policies), which is not necessarily true in reality. For example, it is well known that population behaviors (e.g., awareness of conditions relating to public health, compliance to policies, etc.) vary over time, which can contribute to changes in transmission rates. Moreover, the reporting error associated with the daily confirmed cases (i.e., $D_{t_{p}-1}$ in eq. (1) and the accumulated prediction error in $\hat{r}_{t+k}$ can degenerate the predictions of the daily cases. 
Preprint.

Hence, we introduce a calibration step that uses the data in a short window immediately preceding the forecast window (see Figure 1) to make adjustments. This step makes a reasonable assumption that the relationships between the transmission rate variable and its predictors remain unchanged over a short time period composed of the calibration and forecast windows. Assume prediction should start at time $t_{p}$, we use the time window $\left[t_{p-k}, t_{p-1}\right]$ to linearly calibrate the model trained by using the data up to $t_{p-1}$ as

$$
\log \tilde{D}_{t_{c}}=a \sum_{m=t_{p}-k}^{t_{c}} \log \hat{r}_{m}+b+\log D_{t_{p}-k-1}
$$

where $t_{c} \in\left[t_{p-k}, t_{p-1}\right], \hat{r}_{m}$ is the output of the pre-calibrated model, and $a \& b$ are two calibration parameters. The hyper-parameter $k$ controls the attention span of the calibration step. A small $k$ direct the calibration step to focus on short-term epidemic trends, and vice versa. The parameter $a$ accounts for the time-changing relationship between the transmission rate and its predictors, and $b$ accounts for both the uncertainty associated with $D_{t_{p}-k-1}$ and the error in forecasting $r_{t}$. These two parameters can be solved by optimizing

$$
\begin{aligned}
a^{*}, b^{*}=\underset{a, b}{\operatorname{argmin}} & \sum_{t_{c}=t_{p}-k}^{t_{p-1}}\left[\log \tilde{D}_{t_{c}}-\log D_{t_{c}}\right]^{2} \\
\text { s.t. } & |a-1| \leq \delta
\end{aligned}
$$

where $\delta>0$ controls the maximal scaling effect to prevent numerical instability in estimating $a^{*}$ due to the large uncertainties associated with reports of daily cases. We set $\delta=0.01$ in our experiments. After calibration, the prediction at $t \geq t_{p}$ should be calculated as $\log \hat{D}_{t}=\log \tilde{D}_{t_{p-1}}+$ $a \sum_{m=t_{p}}^{t} \log \hat{r}_{m}$, where $\hat{r}_{m}$ is the $\Delta t$-step-ahead prediction made by the pre-calibrated model. 
Preprint.

\section{B AdDitional RESUlts}
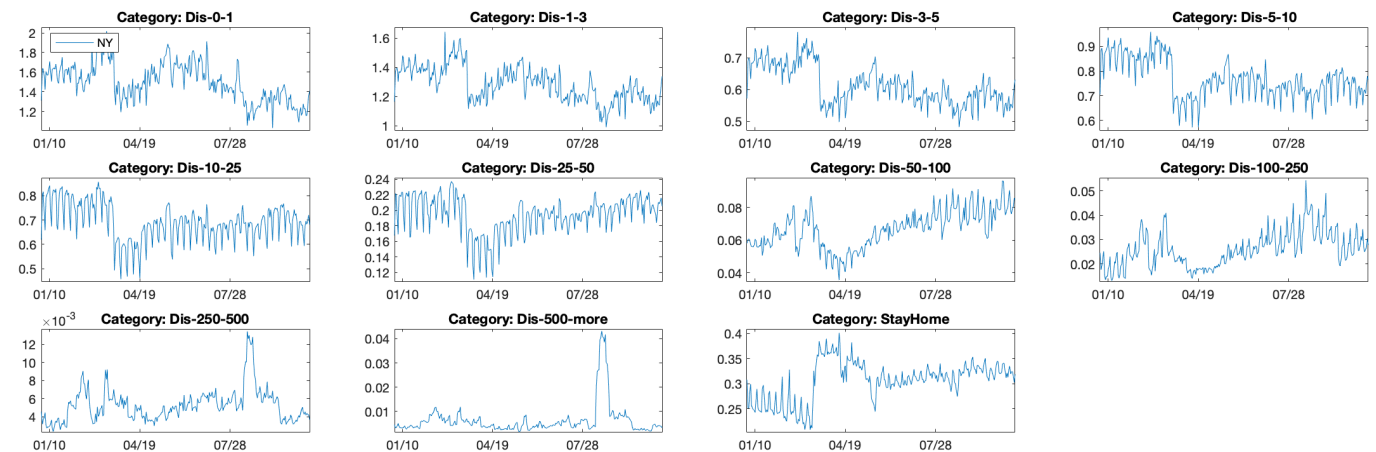

Figure 6: The normalized mobility data of the New York state. There are 11 categories based on the travel distances. The normalization procedure is explained in Section A.1.
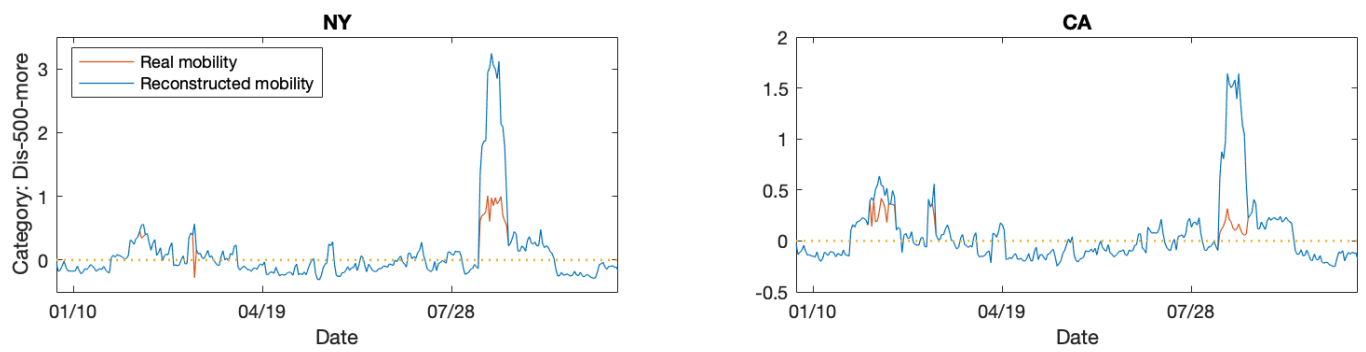

Figure 7: There is a national spike of long-distance travels (the Dis $>500$ mobility category) in midAugust, which might be associated with the starts of schools/universities. The data of two states (NY and CA) are shown as examples. The abnormal mobility samples are "corrected" using Principal Component Analysis as described in Section A.1

Table 1: Regression Table on baseline transmission rates

\begin{tabular}{lrrrr} 
& Estimate & \multicolumn{1}{c}{ SE } & tStat & \multicolumn{1}{c}{ pValue } \\
\hline & & & & \\
(Intercept) $^{*}$ & 0.63678 & 0.20508 & 3.1051 & 0.0032527 \\
Top-density & 0.013506 & 0.006005 & 2.2492 & 0.029331 \\
Adults-35-54 & 1.4687 & 0.8757 & 1.6771 & 0.1003 \\
Hispanic-Or-Latino & 0.21254 & 0.080443 & 2.6421 & 0.011223 \\
AmericanIndian-Or-AlaskaNative & -0.21076 & 0.14421 & -1.4614 & 0.1507 \\
\hline
\end{tabular}

${ }^{*}$ This is the intercept of regression on demographics.

Number of observations: 51, Error degrees of freedom: 46

Root Mean Squared Error: 0.0519

R-squared: 0.421, Adjusted R-Squared: 0.391

F-statistic vs. constant model: $5.34, \mathrm{p}$-value $=1.83 \mathrm{e}-05$ 
Preprint.

\section{It is made available under a CC-BY-NC-ND 4.0 International license .}

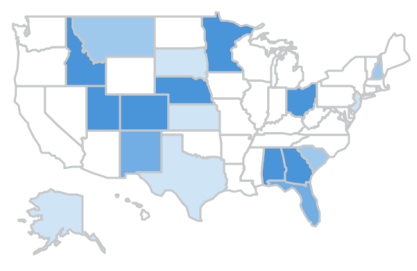

(a) Dis-1-3

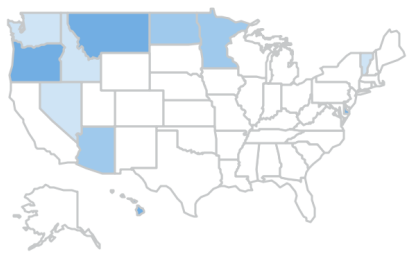

(d) Dis-10-25

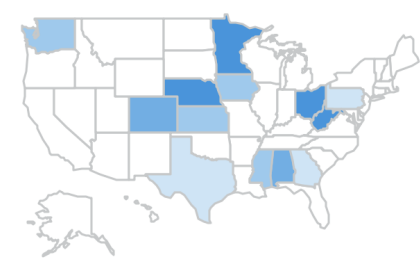

(b) Dis-3-5

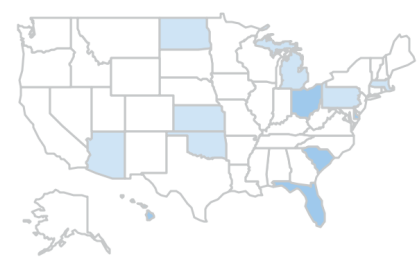

(e) Dis-25-50

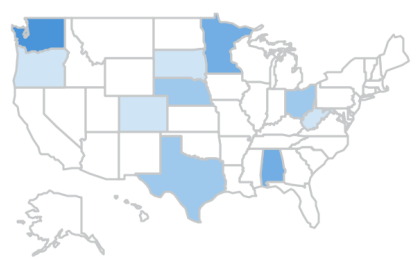

(c) Dis-5-10

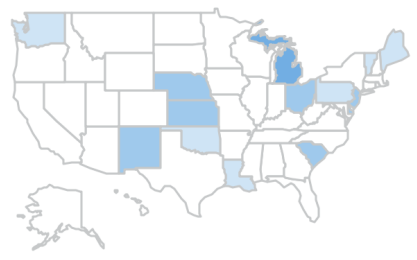

(f) Dis-50-100

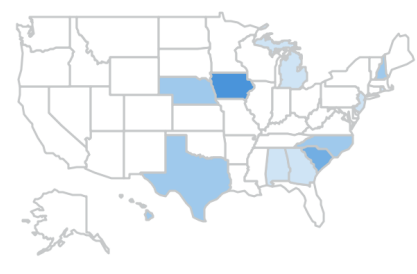

(g) Dis-100-250

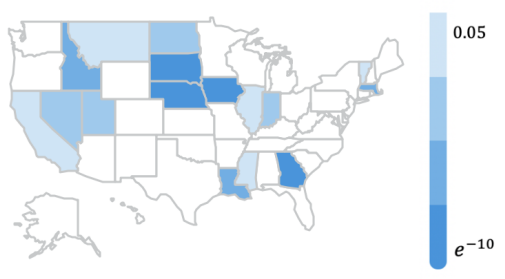

(h) Dis-250-500

Figure 8: The regression $p$-value heatmaps of the mobility data categories.

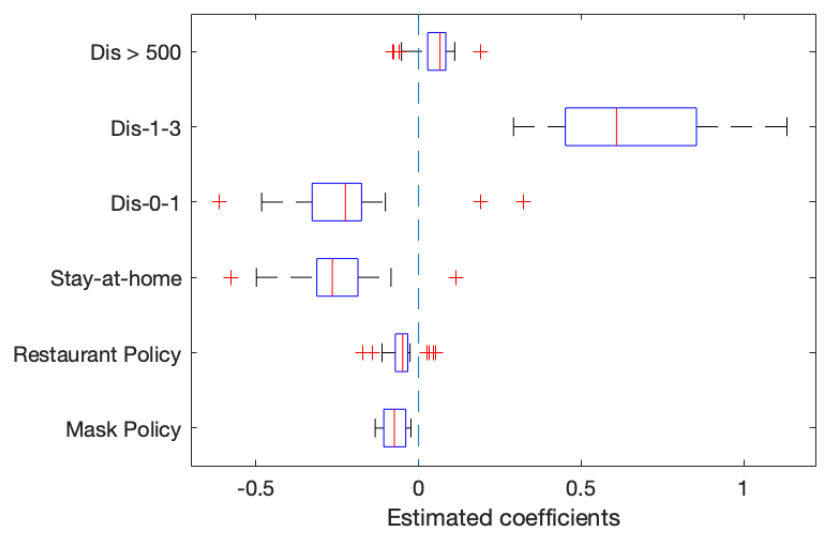

Figure 9: The most generic significant factors identified by our approach (see Figure 4(a) as well as their descriptive statistics of estimated coefficients, according to 51 state-level regression models. Only coefficients with significant level of 0.05 are included in the box plot. The coefficients of Mask Policy, Restaurant Policy, Stay-at-home and Dis-0-1 almost take negative values, showing stable negative correlations with the transmissions rates and indicating that they help prevent the spread of COVID-19. In contrast, the coefficients of Dis-1-3 and Dis $>500$ almost take positive values, showing stable positive correlations with the transmissions rates and indicating that frequent short-distance travels and cross-state travels help promote the spread. 
Preprint.
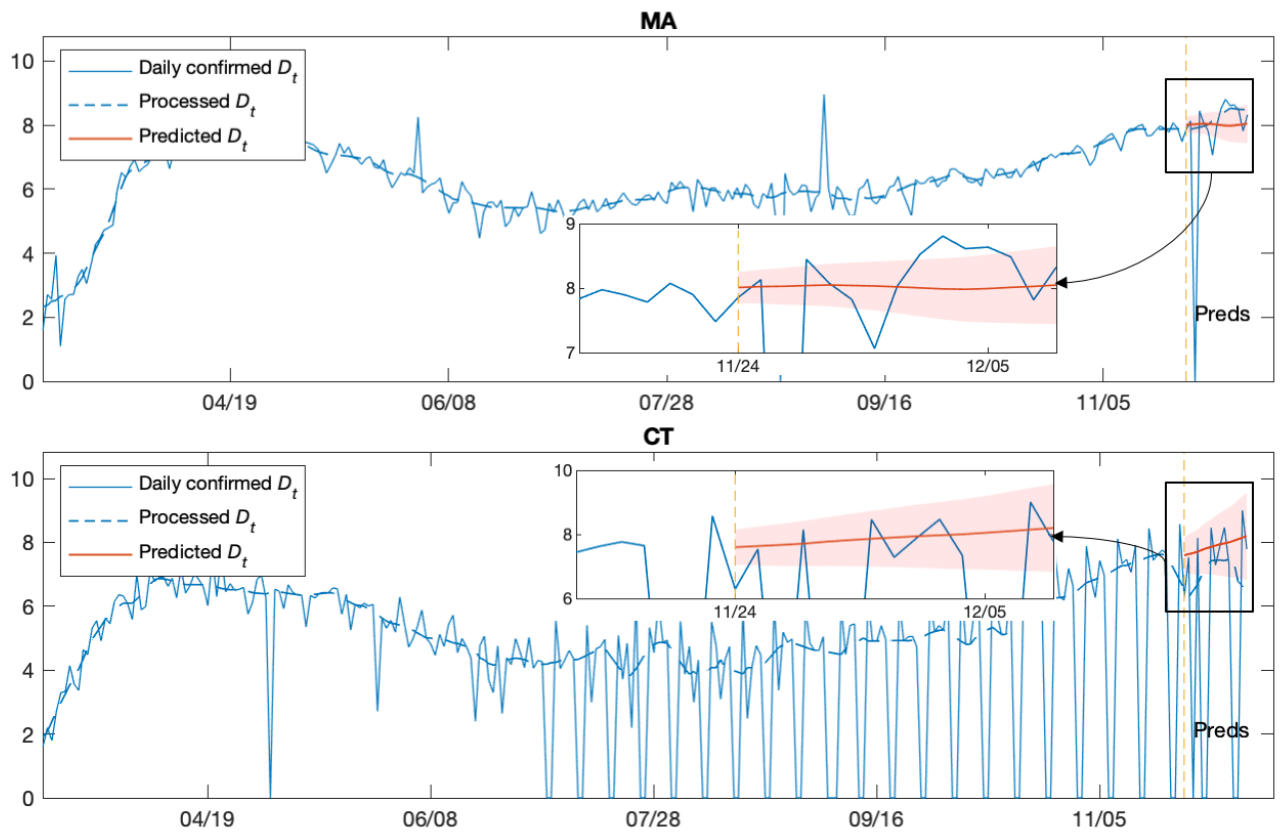

Figure 10: Two representative cases show that the trained models can forecast the trends very well even though they have relatively large prediction nRMSE and RALE: MA (nRMSE $=0.0394$, RALE $=0.0253)$ and CT $(\mathrm{nRMSE}=0.1353$, RALE $=0.1216)$. These large $\mathrm{nRMSE} / \mathrm{RALE}$ values are due to the delays or skips in reporting the COVID-19 daily confirmed cases by the corresponding states. These results indeed indicate the robustness and reliability of our approach. The $x$-axis indicate time. The $y$-axes indicate the logarithm of the daily confirmed cases. The yellow dash vertical lines indicate the starts of the prediction periods. The blowouts highlight the predictions. The red shaded areas indicate the $95 \%$ confidence intervals. 
Table 2: The nRMSE and RALE values of the 3-, 7-, 10-, 14-day state-level predictions.

\begin{tabular}{|c|c|c|c|c|c|c|c|c|}
\hline & \multicolumn{4}{|c|}{ nRMSE } & \multicolumn{4}{|c|}{ RALE } \\
\hline State & 3 days & 7 days & 10 days & 14 days & 3 days & 7 days & 10 days & 14 days \\
\hline AK & 0.0043 & 0.0074 & 0.0067 & 0.0112 & 0.0036 & 0.0066 & 0.0060 & 0.0092 \\
\hline AL & 0.0289 & 0.0282 & 0.0235 & 0.0212 & 0.0284 & 0.0271 & 0.0204 & 0.0187 \\
\hline AR & 0.0433 & 0.0410 & 0.0345 & 0.0294 & 0.0422 & 0.0390 & 0.0246 & 0.0139 \\
\hline AZ & 0.0104 & 0.0217 & 0.0301 & 0.0352 & 0.0068 & 0.0180 & 0.0256 & 0.0314 \\
\hline CA & 0.0042 & 0.0062 & 0.0179 & 0.0278 & 0.0041 & 0.0005 & 0.0095 & 0.0192 \\
\hline $\mathrm{CO}$ & 0.0665 & 0.0831 & 0.0943 & 0.1108 & 0.0662 & 0.0817 & 0.0920 & 0.1069 \\
\hline CT & 0.1779 & 0.1219 & 0.1041 & 0.1353 & 0.1766 & 0.1131 & 0.0978 & 0.1216 \\
\hline DC & 0.0028 & 0.0135 & 0.0470 & 0.0800 & 0.0028 & 0.0028 & 0.0256 & 0.0549 \\
\hline DE & 0.0253 & 0.0273 & 0.0294 & 0.0340 & 0.0253 & 0.0272 & 0.0290 & 0.0331 \\
\hline FL & 0.0192 & 0.0181 & 0.0169 & 0.0224 & 0.0187 & 0.0175 & 0.0162 & 0.0208 \\
\hline GA & 0.0298 & 0.0255 & 0.0317 & 0.0396 & 0.0293 & 0.0203 & 0.0014 & 0.0150 \\
\hline HI & 0.0306 & 0.0505 & 0.0486 & 0.0417 & 0.0284 & 0.0468 & 0.0459 & 0.0380 \\
\hline IA & 0.0706 & 0.0865 & 0.0933 & 0.1139 & 0.0698 & 0.0851 & 0.0918 & 0.1096 \\
\hline ID & 0.0110 & 0.0079 & 0.0084 & 0.0087 & 0.0110 & 0.0069 & 0.0021 & 0.0009 \\
\hline IL & 0.0287 & 0.0326 & 0.0296 & 0.0282 & 0.0281 & 0.0320 & 0.0288 & 0.0275 \\
\hline IN & 0.0365 & 0.0450 & 0.0460 & 0.0492 & 0.0359 & 0.0441 & 0.0453 & 0.0484 \\
\hline KS & 0.0534 & 0.0424 & 0.0446 & 0.0399 & 0.0529 & 0.0365 & 0.0103 & 0.0048 \\
\hline KY & 0.0089 & 0.0164 & 0.0202 & 0.0193 & 0.0080 & 0.0075 & 0.0134 & 0.0143 \\
\hline LA & 0.1211 & 0.0977 & 0.0820 & 0.0755 & 0.1208 & 0.0938 & 0.0758 & 0.0710 \\
\hline MA & 0.0157 & 0.0158 & 0.0318 & 0.0394 & 0.0155 & 0.0028 & 0.0135 & 0.0253 \\
\hline MD & 0.0149 & 0.0159 & 0.0134 & 0.0116 & 0.0148 & 0.0156 & 0.0115 & 0.0067 \\
\hline ME & 0.0153 & 0.0147 & 0.0201 & 0.0305 & 0.0152 & 0.0146 & 0.0187 & 0.0267 \\
\hline MI & 0.0213 & 0.0178 & 0.0193 & 0.0208 & 0.0211 & 0.0174 & 0.0189 & 0.0204 \\
\hline MN & 0.0310 & 0.0470 & 0.0596 & 0.0771 & 0.0308 & 0.0448 & 0.0559 & 0.0710 \\
\hline MO & 0.0463 & 0.0534 & 0.0526 & 0.0536 & 0.0457 & 0.0528 & 0.0522 & 0.0533 \\
\hline MS & 0.0153 & 0.0202 & 0.0298 & 0.0326 & 0.0134 & 0.0066 & 0.0181 & 0.0241 \\
\hline MT & 0.0242 & 0.0263 & 0.0332 & 0.0411 & 0.0241 & 0.0260 & 0.0320 & 0.0390 \\
\hline $\mathrm{NC}$ & 0.0146 & 0.0212 & 0.0187 & 0.0159 & 0.0137 & 0.0201 & 0.0168 & 0.0133 \\
\hline ND & 0.0231 & 0.0260 & 0.0332 & 0.0364 & 0.0231 & 0.0256 & 0.0318 & 0.0348 \\
\hline $\mathrm{NE}$ & 0.0314 & 0.0412 & 0.0515 & 0.0810 & 0.0310 & 0.0402 & 0.0487 & 0.0714 \\
\hline $\mathrm{NH}$ & 0.0271 & 0.0189 & 0.0157 & 0.0143 & 0.0266 & 0.0166 & 0.0121 & 0.0055 \\
\hline NJ & 0.0260 & 0.0331 & 0.0331 & 0.0322 & 0.0257 & 0.0324 & 0.0325 & 0.0317 \\
\hline NM & 0.0089 & 0.0206 & 0.0225 & 0.0294 & 0.0069 & 0.0177 & 0.0203 & 0.0262 \\
\hline NV & 0.0159 & 0.0240 & 0.0286 & 0.0338 & 0.0155 & 0.0228 & 0.0271 & 0.0319 \\
\hline NY & 0.0090 & 0.0072 & 0.0102 & 0.0105 & 0.0090 & 0.0053 & 0.0007 & 0.0036 \\
\hline $\mathrm{OH}$ & 0.0322 & 0.0417 & 0.0448 & 0.0480 & 0.0319 & 0.0408 & 0.0439 & 0.0470 \\
\hline OK & 0.0303 & 0.0530 & 0.0546 & 0.0518 & 0.0279 & 0.0488 & 0.0516 & 0.0497 \\
\hline OR & 0.0058 & 0.0142 & 0.0148 & 0.0132 & 0.0049 & 0.0123 & 0.0134 & 0.0116 \\
\hline PA & 0.0423 & 0.0512 & 0.0519 & 0.0525 & 0.0420 & 0.0504 & 0.0511 & 0.0518 \\
\hline RI & 0.0592 & 0.0551 & 0.0596 & 0.1206 & 0.0591 & 0.0549 & 0.0592 & 0.0992 \\
\hline $\mathrm{SC}$ & 0.0097 & 0.0098 & 0.0275 & 0.0484 & 0.0093 & 0.0020 & 0.0126 & 0.0315 \\
\hline SD & 0.0660 & 0.0839 & 0.0900 & 0.1130 & 0.0649 & 0.0820 & 0.0883 & 0.1075 \\
\hline TN & 0.0160 & 0.0156 & 0.0144 & 0.0127 & 0.0160 & 0.0155 & 0.0143 & 0.0120 \\
\hline TX & 0.0278 & 0.0241 & 0.0229 & 0.0264 & 0.0278 & 0.0239 & 0.0228 & 0.0257 \\
\hline UT & 0.0423 & 0.0332 & 0.0299 & 0.0281 & 0.0423 & 0.0290 & 0.0140 & 0.0029 \\
\hline VA & 0.0042 & 0.0157 & 0.0148 & 0.0172 & 0.0011 & 0.0119 & 0.0111 & 0.0017 \\
\hline VT & 0.0635 & 0.0589 & 0.0605 & 0.0747 & 0.0632 & 0.0549 & 0.0199 & 0.0165 \\
\hline WA & 0.0073 & 0.0058 & 0.0076 & 0.0177 & 0.0071 & 0.0018 & 0.0019 & 0.0098 \\
\hline WI & 0.0328 & 0.0403 & 0.0418 & 0.0423 & 0.0325 & 0.0397 & 0.0412 & 0.0418 \\
\hline WV & 0.0439 & 0.0485 & 0.0508 & 0.0535 & 0.0434 & 0.0480 & 0.0503 & 0.0531 \\
\hline WY & 0.0681 & 0.0933 & 0.1049 & 0.1285 & 0.0671 & 0.0902 & 0.1014 & 0.1224 \\
\hline
\end{tabular}

